\title{
Flow of non-Newtonian Fluids in Converging-Diverging Rigid Tubes
}

Taha Sochi*

October 30, 2013

*University College London, Department of Physics \& Astronomy, Gower Street, London, WC1E 6BT. Email: t.sochi@ucl.ac.uk. 


\section{Contents}

$\begin{array}{ll}\text { Contents } & 2\end{array}$

$\begin{array}{ll}\text { Abstract } & 3\end{array}$

1 Introduction 4

2 Investigated non-Newtonian Fluids 5

2.1 Ellis Model . . . . . . . . . . . . . . . . . . 6

2.2 Herschel-Bulkley Model . . . . . . . . . . . . . . . . . . . 7

3 Method $\quad 8$

4 Implementation and Results $\quad 10$

5 Tests and Validations $\quad 21$

6 Computational Issues and Comparison 24

$\begin{array}{llr}7 & \text { Conclusions } & 26\end{array}$

$\begin{array}{lr}\text { Nomenclature } & 28\end{array}$

$\begin{array}{ll}\text { References } & 30\end{array}$ 


\section{Abstract}

A residual-based lubrication method is used in this paper to find the flow rate and pressure field in converging-diverging rigid tubes for the flow of timeindependent category of non-Newtonian fluids. Five converging-diverging prototype geometries were used in this investigation in conjunction with two fluid models: Ellis and Herschel-Bulkley. The method was validated by convergence behavior sensibility tests, convergence to analytical solutions for the straight tubes as special cases for the converging-diverging tubes, convergence to analytical solutions found earlier for the flow in converging-diverging tubes of Newtonian fluids as special cases for non-Newtonian, and convergence to analytical solutions found earlier for the flow of power-law fluids in converging-diverging tubes. A brief investigation was also conducted on a sample of diverging-converging geometries. The method can in principle be extended to the flow of viscoelastic and thixotropic/rheopectic fluid categories. The method can also be extended to geometries varying in size and shape in the flow direction, other than the perfect cylindrically-symmetric converging-diverging ones, as long as characteristic flow relations correlating the flow rate to the pressure drop on the discretized elements of the lubrication approximation can be found. These relations can be analytical, empirical and even numerical and hence the method has a wide applicability range.

Keywords: fluid mechanics; non-Newtonian fluids; Ellis; Herschel-Bulkley; yieldstress; power-law; Bingham; converging-diverging tubes; diverging-converging tubes; non-linear systems. 


\section{Introduction}

The flow of fluids in general and non-Newtonian in particular through conduits with varying cross sectional size and/or shape is commonplace in natural and industrial flow systems such as filtration and refinement devices in the chemical industries and biological fluid transportation networks like blood circulation vessels and air respiration pathways [1-8]. Modeling such flows is also needed for analyzing fluid transportation through porous media where the irregularly-shaped pores and throats are usually described by geometrically-simple flow conduits with varying cross sections in the flow direction [9-18]. In this respect, pore-scale network modeling is the main candidate and the most natural approach to accommodate such flow-structure features [19-24]. The effect of varying cross section in the flow direction can be particularly important for modeling certain flow phenomena such as extensional flows, viscoelastic effects and yield-stress dynamics which are essential for various scientific and technological purposes like enhanced oil recovery as well as many other applications [25-36].

Several methods have been used in the past to model and simulate such flows; these include numerical methods like finite element, finite difference and stochastic algorithms [10-12, 37-39], as well as analytically-based methods [1, 2, 40-42]. Most previous attempts employ complex mathematical and computational techniques, which may be unwanted due to difficulties in implementation and verification, high computational costs, computational complexities like convergence difficulties, as well as susceptibility to errors and bugs. Also, many of the previous investigations are relevant only to special cases of fluid or flow or conduit geometry with a subsequent limited use. Therefore, developing a simple, general and robust method to investigate such flows is highly desired.

In this paper we use a residual-based lubrication approximation method to find the flow of non-Newtonian fluids through rigid tubes with converging-diverging 
and diverging-converging shapes in the axial direction. The method is based on discretizing the flow conduits in the axial dimension into ring-like elements on which non-Newtonian characteristic flow relations derived for conduits with a fixed axial geometry can apply. The formulation in this paper is limited to the timeindependent category of the non-Newtonian fluids; although the method in principle is capable of being extended to history-dependent fluids, i.e. viscoelastic [25] and thixotropic/rheopectic. Two widely used non-Newtonian fluid models, Ellis and Herschel-Bulkley, are examined in this study as prototypes for the timeindependent models to which this method applies. These two fluids can be used to model diverse non-Newtonian rheological phenomena such as shear-thinning, shearthickening, and yield stress as well as Newtonian flow as a special case. These fluid models are commonly used for describing the time-independent non-Newtonian rheology especially in the industrial applications such as polymer manufacturing and processing and oil recovery [43, 44].

Although the present paper investigates the flow of non-Newtonian fluids in certain axi-symmetric geometries with converging and diverging features, the method is more general and can be used with other geometries whose cross section varies in size and/or shape in the flow direction. The only condition for the applicability of the method is the availability of analytic, empirical or numerical [45] relations that correlate the flow rate in the discretized elements to the pressure drop across these elements.

\section{Investigated non-Newtonian Fluids}

In this section we present a general background about the bulk rheology and flow relations for the two investigated non-Newtonian fluids: Ellis and Herschel-Bulkley. 


\subsection{Ellis Model}

This is a three-parameter model that is widely employed to describe the flow of time-independent shear-thinning yield-free non-Newtonian fluids. The model is known for being successful in describing the bulk and in situ rheologies of a wide range of polymeric solutions. It is particularly used as a replacement for the powerlaw model due to its superiority in matching experimental data. The model is characterized by a single low-shear-rate Newtonian plateau with the absence of a high-shear-rate one and hence it may better match the observations in low and medium shear-rate regimes than in the high shear-rate regimes.

An attractive feature of the Ellis model is the availability of an analytical expression that links the flow rate to the pressure drop for the flow in cylindrical constant-radius tubes. This feature makes rheological modeling with Ellis more accurate and convenient than with some other models, such as Carreau, which have no such analytical expressions although these models may be as good as or even superior to Ellis. The advantage of having a closed-form analytical flow characterization relation is obvious especially for some numerical techniques like pore-scale network modeling.

According to the Ellis model, the fluid viscosity $\mu$ as a function of the shearstress is given by the following expression [22, 46-50]

$$
\mu=\frac{\mu_{o}}{1+\left(\frac{\tau}{\tau_{1 / 2}}\right)^{\alpha-1}}
$$

where $\mu_{o}$ is the zero-shear-rate viscosity, $\tau$ is the shear stress, $\tau_{1 / 2}$ is the shear stress at which $\mu=\frac{\mu_{o}}{2}$ and $\alpha$ is a flow behavior index in this model.

For Ellis fluids, the volumetric flow rate as a function of the pressure drop in a circular cylindrical tube with a constant radius over its length assuming a laminar incompressible flow with no slip at the tube wall [51] is given by the following 
equation $[22,52]$

$$
Q=\frac{\pi R^{4} \Delta p}{8 L \mu_{o}}\left[1+\frac{4}{\alpha+3}\left(\frac{R \Delta p}{2 L \tau_{1 / 2}}\right)^{\alpha-1}\right]
$$

where $R$ is the tube radius, $\Delta p$ is the pressure drop over the tube length and $L$ is the tube length. The derivation of this expression is given in $[22,52]$.

\subsection{Herschel-Bulkley Model}

This is a three-parameter model that can describe Newtonian and large group of time-independent non-Newtonian fluids. According to the Herschel-Bulkley model, the shear stress as a function of the shear rate is given by the following relation $[22,50,53]$

$$
\tau=\tau_{o}+C \dot{\gamma}^{n} \quad\left(\tau>\tau_{o}\right)
$$

where $\tau$ is the shear stress, $\tau_{o}$ is the yield-stress above which the substance starts to flow, $C$ is the consistency coefficient, $\dot{\gamma}$ is the shear rate and $n$ is the flow behavior index. The Herschel-Bulkley model reduces to the power-law, or Ostwald-de Waele model, when the yield-stress is zero, and to the Bingham plastic model when the flow behavior index is unity. It also emulates the Newtonian fluids when both the yield-stress is zero and the flow behavior index is unity [54].

The Herschel-Bulkley model contains six classes: (a) shear-thinning without yield-stress $\left[n<1.0, \tau_{o}=0\right]$ which is the power-law fluid in shear-thinning mode, (b) shear-thinning with yield-stress $\left[n<1.0, \tau_{o}>0\right]$, (c) Newtonian $\left[n=1.0, \tau_{o}=\right.$ 0], (d) Bingham plastic $\left[n=1.0, \tau_{o}>0\right]$, (e) shear-thickening (dilatant) without yield-stress $\left[n>1.0, \tau_{o}=0\right]$, and (f) shear-thickening with yield-stress $[n>$ $\left.1.0, \tau_{o}>0\right]$.

For Herschel-Bulkley fluids, the volumetric flow rate as a function of the pressure drop in a circular cylindrical tube with a fixed radius assuming a laminar 
incompressible flow with no wall slip $[51,55]$ is given by the following relation $[22,52,53]:$

$$
\begin{array}{ll}
Q=0 & \left(\tau_{w} \leq \tau_{o}\right) \\
Q=\frac{8 \pi}{C^{\frac{1}{n}}}\left(\frac{L}{\Delta p}\right)^{3}\left(\tau_{w}-\tau_{o}\right)^{1+\frac{1}{n}}\left[\frac{\left(\tau_{w}-\tau_{o}\right)^{2}}{3+1 / n}+\frac{2 \tau_{o}\left(\tau_{w}-\tau_{o}\right)}{2+1 / n}+\frac{\tau_{o}^{2}}{1+1 / n}\right] & \left(\tau_{w}>\tau_{o}\right)
\end{array}
$$

where $\tau_{w}$ is the shear stress at the tube wall which is given by $\tau_{w}=\frac{\Delta p R}{2 L}$, while the meaning of the other symbols have already been given. The derivation of this expression can be found in [22, 52].

\section{Method}

According to the residual-based lubrication approach, which is proposed in the present paper to find the flow rate and pressure field in converging-diverging tubes for the flow of time-independent non-Newtonian fluids, the tube is discretized in the axial dimension into ring-like elements. Each one of these elements is treated as a constant-radius single tube, where the radius of the element is taken as the average of its inlet and outlet radii, to which Equations 2 and 4 apply. From this discretized form of the flow conduit, a system of non-linear simultaneous equations, which are based on the mass conservation residual plus boundary conditions, is formed.

For a discretized tube consisting of $(N-1)$ elements, there are $N$ nodes: $(N-2)$ internal nodes and two boundary nodes. Each one of these nodes is characterized by a well-defined axial pressure according to the one-dimensional flow model. Also for the internal nodes, and because of the assumption of flow incompressibility, the total sum of the volumetric flow rate, which is signed $(+/-)$ according to its direction as being toward or away from the given node, is zero due to the lack of sources and sinks at the node, and hence $(N-2)$ residual functions whose essence is the vanishing of the net flow at the internal nodes are formed. These residual 
equations are coupled with two given boundary conditions at the inlet and outlet nodes to form a system of $N$ simultaneous equations.

A typical method for solving such a system is to use an iterative non-linear solution scheme such as Newton-Raphson method where an initial guess for the internal nodal pressures is proposed and used in conjunction with the Jacobian matrix of the residual functions to find the pressure perturbation vector which is then used to adjust the values of the internal nodal pressure. This iterative process is repeated until a convergence condition, which is usually based on the size of the residual norm, is reached.

In more formal terms, the process is based on solving the following matrix equation iteratively

$$
\mathbf{J} \Delta \mathbf{p}=-\mathbf{r}
$$

where $\mathbf{J}$ is the Jacobian matrix, $\mathbf{p}$ is the vector of variables which represent the pressure values at the boundary and internal nodes, and $\mathbf{r}$ is the vector of residuals which, for the internal nodes, is based on the continuity of the volumetric flow rate as given by

$$
f_{j}=\sum_{i=1}^{m} Q_{i}=0
$$

where $m$ is the number of discretized elements connected to node $j$ which is two in this case, and $Q_{i}$ is the signed volumetric flow rate in element $i$ as characterized by Equations 2 and 4 . Equation 5 is then solved in each iteration for $\Delta \mathbf{p}$ which is then used to update $\mathbf{p}$. The convergence will be announced when the norm of the residual vector, $\mathbf{r}$, becomes within a predefined tolerated marginal error. More details about this solution scheme can be found in $[38,56,57]$. 


\section{Implementation and Results}

The residual-based lubrication method was implemented in a computer code and flow simulation results were obtained for a wide range of fluid, flow and tube parameters for both Ellis and Herschel-Bulkley fluids. All the simulations reported in the present paper were carried out using evenly-divided discretization meshes. In this investigation we used five cylindrically-symmetric converging-diverging tube geometries, a graphic demonstration of which is shown in Figure 1. The equations that describe the radius dependency on the tube axial coordinate for these five geometries are given in Table 1, while a generic converging-diverging tube profile, demonstrating the coordinate system used in the mathematical formulation of this dependency, is shown in Figure 2.

A sample of the results of these simulations are shown in Figure 3 for Ellis fluids and Figure 4 for Herschel-Bulkley fluids using the five geometries of Table 1. In these figures the tube axial pressure is plotted as a function of the tube axial coordinate for a number of authentic fluids whose bulk rheologies are given in Table 2 for the Ellis fluids and Table 3 for the Herschel-Bulkley fluids. The pressure dependency for the Poiseuille flow of Newtonian fluids having the same zero-shearrate viscosity is also given in these figures for demonstration and comparison. The inclusion of the Poiseuille flow can also serve as a sensibility test; for example in Figure 3 (c) we see a strong similarity in the pressure field and flow rate between the Poiseuille and Ellis flows since the latter has a strong resemblance to the Newtonian at these flow regimes as can be seen from its rheological parameters. Also for the flow represented by Figure 4 (e) we observe the convergence of the Bingham flow rate to the Newtonian flow rate at this high-pressure flow regime which is a sensible trend. Unlike the flow rates of the other figures, the non-Newtonian flow in Figure 4 (e) is lower than the Newtonian flow rate because the non-Newtonian is a Bingham fluid and not a shear-thinning fluid. The effect of the yield-stress in this case is to 
lower the flow rate of Bingham from its Newtonian counterpart, as expected. It should be remarked that in Figures 3 and $4, p_{i}$ and $p_{o}$ represent the inlet and outlet pressures, while $Q_{E}, Q_{H}$ and $Q_{P}$ are the flow rates for Ellis, Herschel-Bulkley and Poiseuille respectively. The plots shown in these figures represent the converged solutions which are obtained with the use of discretization meshes that normally consist of $50-100$ elements.

In Figures 5-9 we present a sample of our results in a different form where we plot the volumetric flow rate as a function of the pressure drop for a number of converging-diverging geometries using a representative sample of Ellis and Herschel-Bulkley fluids. It should be remarked that the residual-based lubrication method can also be used with diverging-converging geometries similar to the ones demonstrated in Figure 10. A sample of the flow simulation results using the latter geometries with a representative sample of Ellis and Herschel-Bulkley fluids is shown in Figures 11-13. In each one of these figures, plots of both axial pressure versus axial coordinate and flow rate versus pressure drop are given. An interesting feature is that the curvature of the pressure field plots in these figures is qualitatively different from that seen in the plots of the converging-diverging geometries, which is a sensible tendency as it reflects the nature of the conduit geometry. 


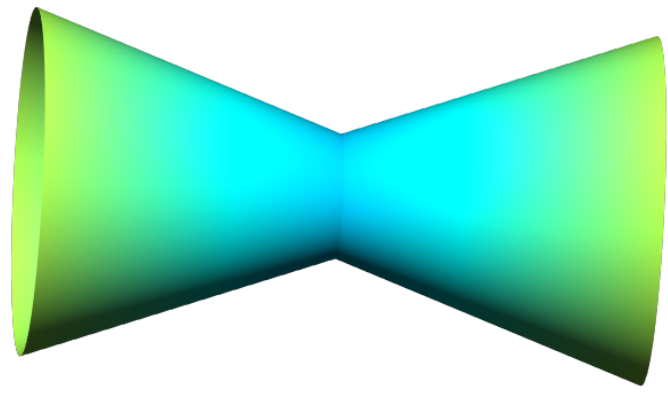

(a) Conic

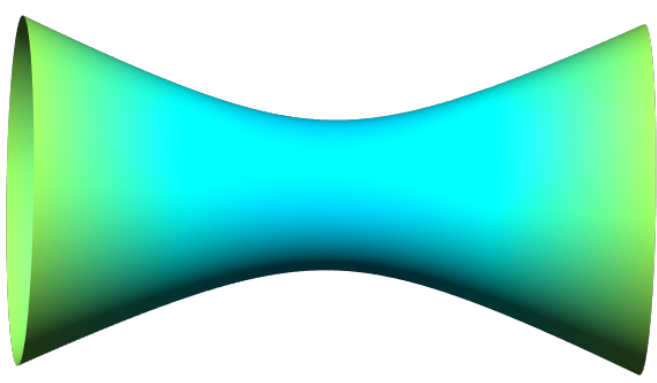

(c) Hyperbolic

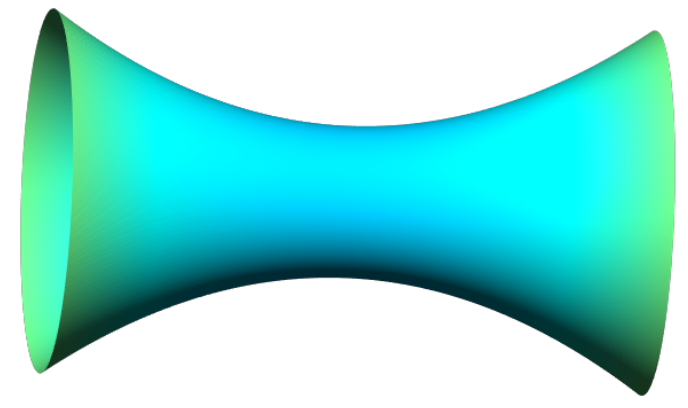

(b) Parabolic

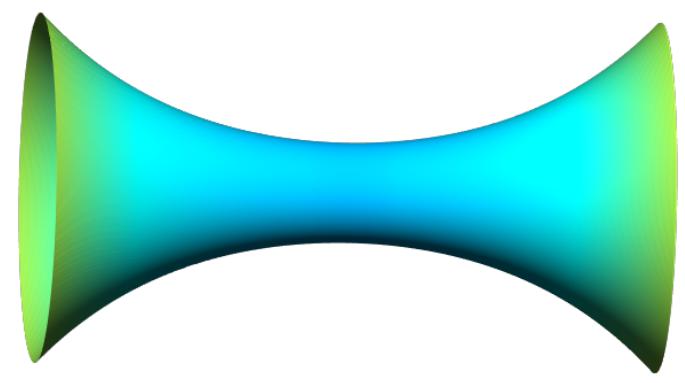

(d) Hyperbolic Cosine

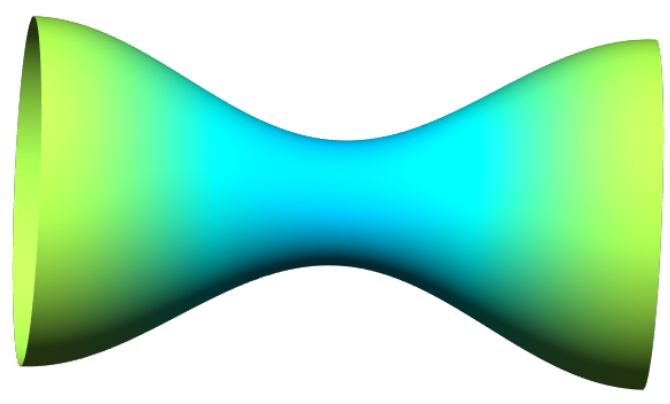

(e) Sinusoidal

Figure 1: A graphic demonstration of the converging-diverging tube geometries used in this investigation. 
Table 1: The correlation between the tube radius $R$ and its axial coordinate $x$ for the five converging-diverging geometries used in this paper. In these equations, $-\frac{L}{2} \leq x \leq \frac{L}{2}$ and $R_{m}<R_{M}$ where $R_{m}$ is the tube minimum radius at $x=0$ and $R_{M}$ is the tube maximum radius at $x= \pm \frac{L}{2}$ as demonstrated in Figure 2.

\begin{tabular}{ll}
\hline Geometry & $R(x)$ \\
\hline Conic & $R_{m}+\frac{2\left(R_{M}-R_{m}\right)}{L}|x|$ \\
Parabolic & $R_{m}+\left(\frac{2}{L}\right)^{2}\left(R_{M}-R_{m}\right) x^{2}$ \\
Hyperbolic & $\sqrt{R_{m}^{2}+\left(\frac{2}{L}\right)^{2}\left(R_{M}^{2}-R_{m}^{2}\right) x^{2}}$ \\
Hyperbolic Cosine & $R_{m} \cosh \left[\frac{2}{L} \operatorname{arccosh}\left(\frac{R_{M}}{R_{m}}\right) x\right]$ \\
Sinusoidal & $\left(\frac{R_{M}+R_{m}}{2}\right)-\left(\frac{R_{M}-R_{m}}{2}\right) \cos \left(\frac{2 \pi x}{L}\right)$ \\
\hline
\end{tabular}

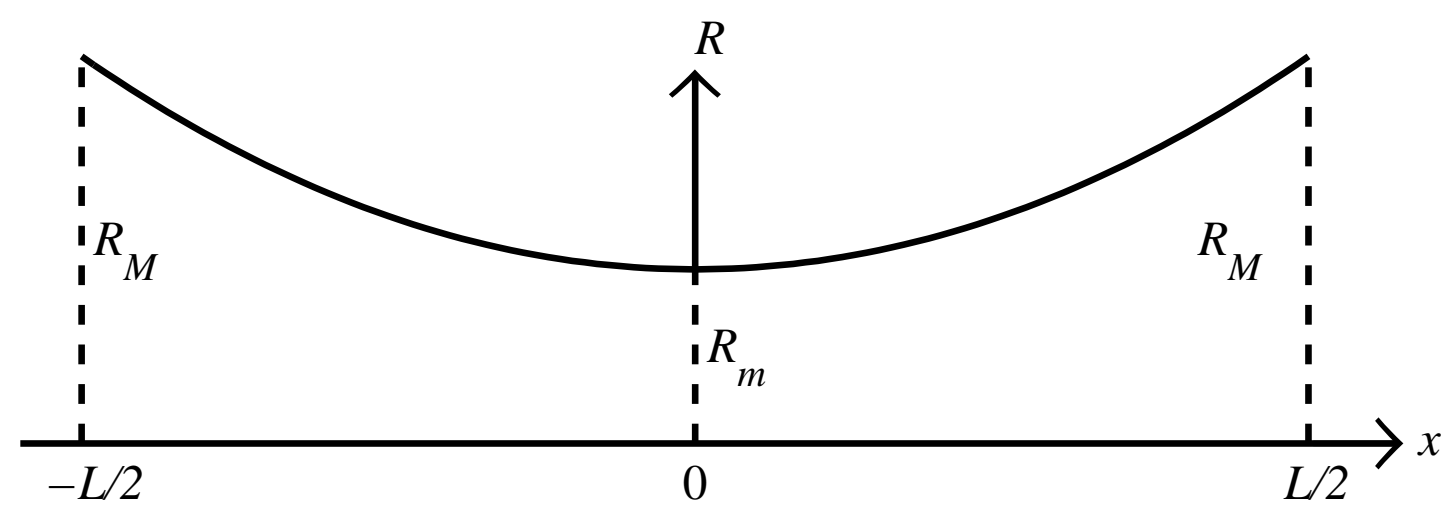

Figure 2: A schematic profile of the converging-diverging tubes that demonstrates the setting of the coordinate system used in the equations correlating the tube radius $R$ to its axial coordinate $x$ as used in Table 1 .

Table 2: Bulk rheology data related to the Ellis fluids used to generate the plots in Figure 3.

\begin{tabular}{lccccc}
\hline & Source & Fluid & $\mu_{o}$ (Pa.s) & $\alpha$ & $\tau_{1 / 2}(\mathrm{~Pa})$ \\
\hline Fig. 3 (a) & Sadowski [58] & $0.4 \%$ Natrosol - 250H & 0.1000 & 1.811 & 2.2 \\
Fig. 3 (b) & Sadowski [58] & $1.4 \%$ Natrosol - 250G & 0.0688 & 1.917 & 59.9 \\
Fig. 3 (c) & Sadowski [58] & 6.0\% Elvanol 72-51 & 0.1850 & 2.400 & 1025.0 \\
Fig. 3 (d) & Park [59] & polyacrylamide 0.50\% & 4.35213 & 2.4712 & 0.7185 \\
Fig. 3 (e) & Park [59] & polyacrylamide 0.05\% & 0.26026 & 2.1902 & 0.3390 \\
\hline
\end{tabular}




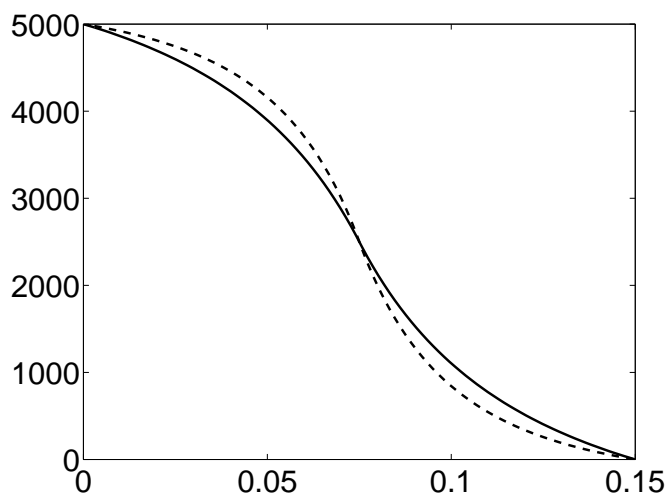

(a) Conic

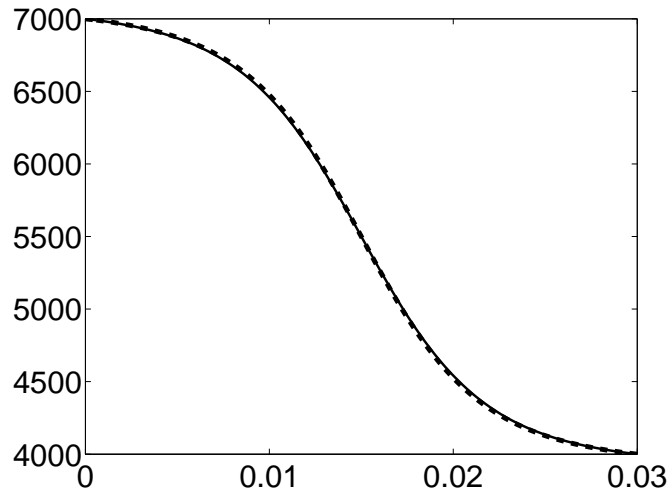

(c) Hyperbolic

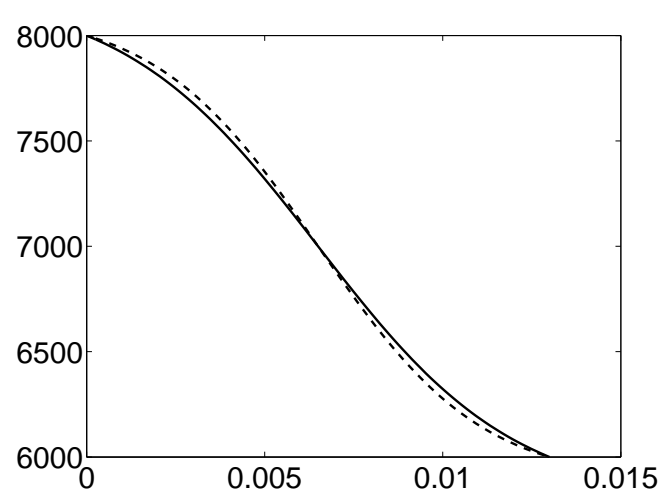

(b) Parabolic

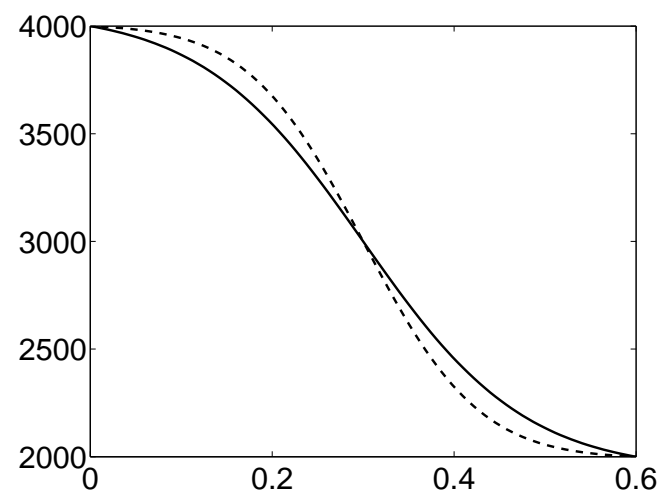

(d) Hyperbolic Cosine

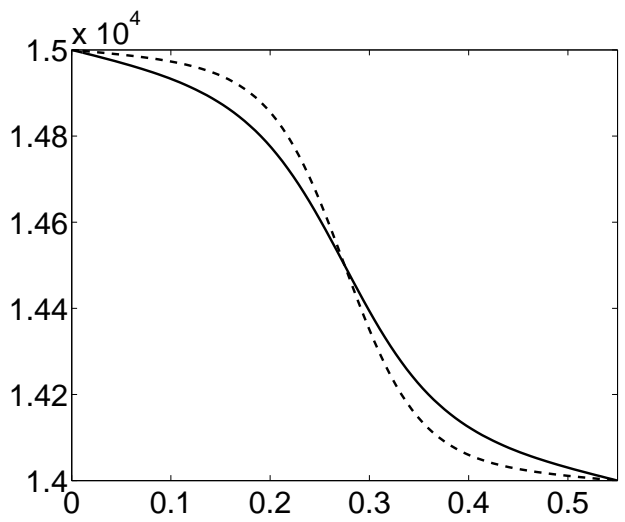

(e) Sinusoidal

Figure 3: Comparing Ellis (solid) to Poiseuille (dashed) models for convergingdiverging rigid tubes of the given geometry: (a) conic with $L=0.15, R_{m}=0.01$, $R_{M}=0.02, p_{i}=5000, p_{o}=0, Q_{E}=0.187942, Q_{P}=0.00452118$ (b) parabolic with $L=0.013, R_{m}=0.0017, R_{M}=0.0025, p_{i}=8000, p_{o}=6000, Q_{E}=3.43037 \times 10^{-5}$, $Q_{P}=1.16241 \times 10^{-5}$ (c) hyperbolic with $L=0.03, R_{m}=0.002, R_{M}=0.004$, $p_{i}=7000, p_{o}=4000, Q_{E}=8.49764 \times 10^{-6}, Q_{P}=8.0162 \times 10^{-6}(\mathrm{~d})$ hyperbolic cosine with $L=0.6, R_{m}=0.04, R_{M}=0.1, p_{i}=4000, p_{o}=2000, Q_{E}=1.8855$, $Q_{P}=0.00184536$ (e) sinusoidal with $L=0.55, R_{m}=0.03, R_{M}=0.07, p_{i}=15000$, $p_{o}=14000, Q_{E}=2.14775, Q_{P}=0.00757797$. All dimensional quantities are in standard SI units. In all five sub-figures, the vertical axis represents the axial pressure in pascals while the horizontal axis represents the tube axial coordinate in meters. The rheological properties of the fluids are given in Table 2. 


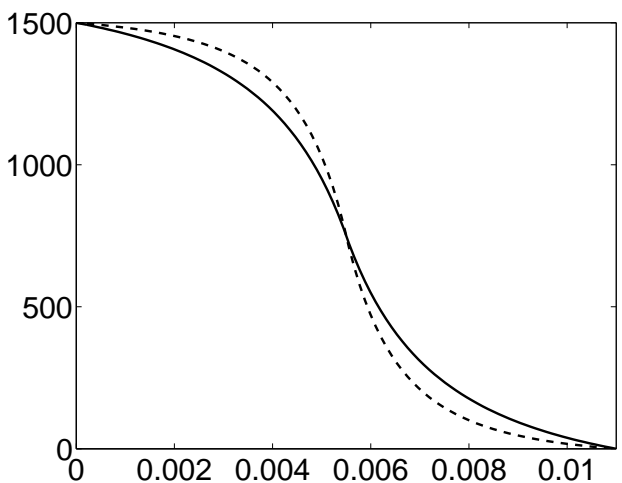

(a) Conic

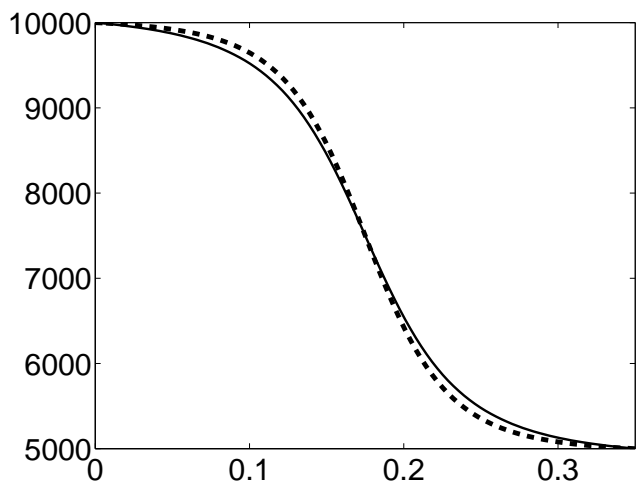

(c) Hyperbolic

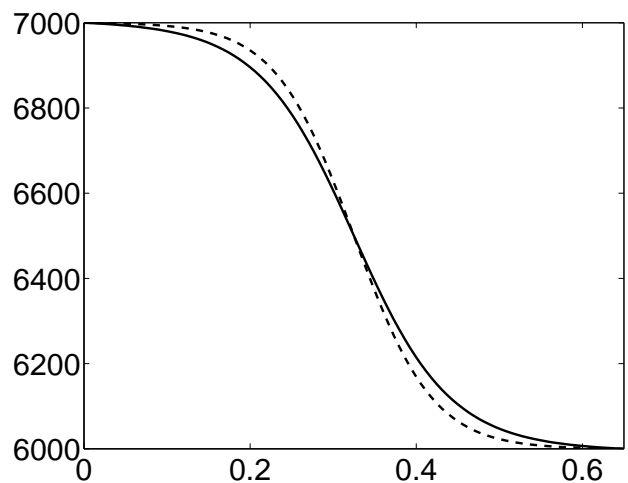

(b) Parabolic

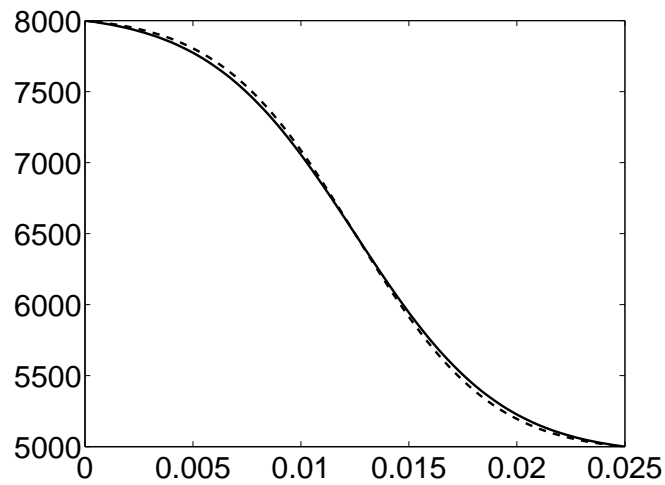

(d) Hyperbolic Cosine

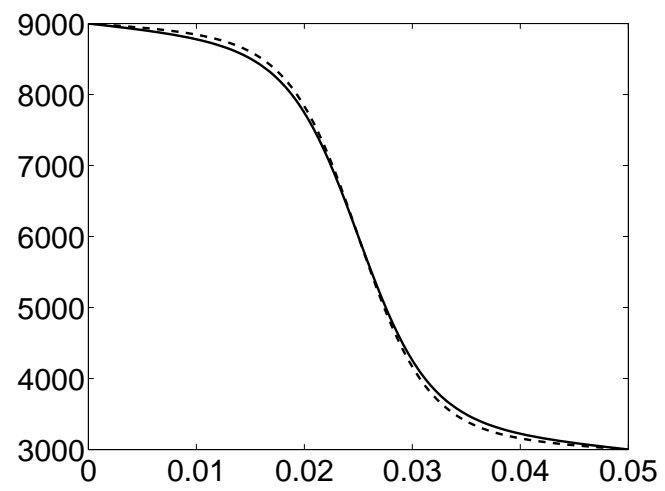

(e) Sinusoidal

Figure 4: Comparing Herschel-Bulkley (solid) to Poiseuille (dashed) models for converging-diverging rigid tubes of the given geometry: (a) conic with $L=0.011$, $R_{m}=0.001, R_{M}=0.0027, p_{i}=1500, p_{o}=0, Q_{H}=4.4286 \times 10^{-4}, Q_{P}=$ $2.49962 \times 10^{-6}$ (b) parabolic with $L=0.65, R_{m}=0.04, R_{M}=0.15, p_{i}=7000$, $p_{o}=6000, Q_{H}=23.9883, Q_{P}=0.252061$ (c) hyperbolic with $L=0.35, R_{m}=0.03$, $R_{M}=0.08, p_{i}=10000, p_{o}=5000, Q_{H}=0.0625224, Q_{P}=0.012097(\mathrm{~d})$ hyperbolic cosine with $L=0.025, R_{m}=0.0025, R_{M}=0.005, p_{i}=8000, p_{o}=5000, Q_{H}=$ $1.90137 \times 10^{-5}, Q_{P}=8.13167 \times 10^{-6}$ (e) sinusoidal with $L=0.05, R_{m}=0.004$, $R_{M}=0.01, p_{i}=9000, p_{o}=3000, Q_{H}=1.84272 \times 10^{-4}, Q_{P}=2.04623 \times 10^{-4}$. All dimensional quantities are in standard SI units. In all five sub-figures, the vertical axis represents the axial pressure in pascals while the horizontal axis represents the tube axial coordinate in meters. The rheological properties of the fluids are given in Table 3. 
Table 3: Bulk rheology data related to the Herschel-Bulkley fluids used to generate the plots in Figure 4.

\begin{tabular}{lccccc}
\hline & Source & Fluid & $C\left(\right.$ Pa.s $\left.^{n}\right)$ & $n$ & $\tau_{o}(\mathrm{~Pa})$ \\
\hline Fig. 4 (a) & Park [59] & $0.50 \%$ PMC 400 & 0.116 & 0.57 & 0.535 \\
Fig. 4 (b) & Park [59] & 0.50\% PMC 25 & 0.021 & 0.63 & 0.072 \\
Fig. 4 (c) & Al-Fariss \& Pinder [60] & waxy oil 4\% & 1.222 & 0.77 & 3.362 \\
Fig. 4 (d) & Al-Fariss \& Pinder [60] & waxy oil 5\% & 0.463 & 0.87 & 3.575 \\
Fig. 4 (e) & Chase \& Dachavijit [61] & Carbopol 941 1.3\% & 0.215 & 1.00 & 28.46 \\
\hline
\end{tabular}

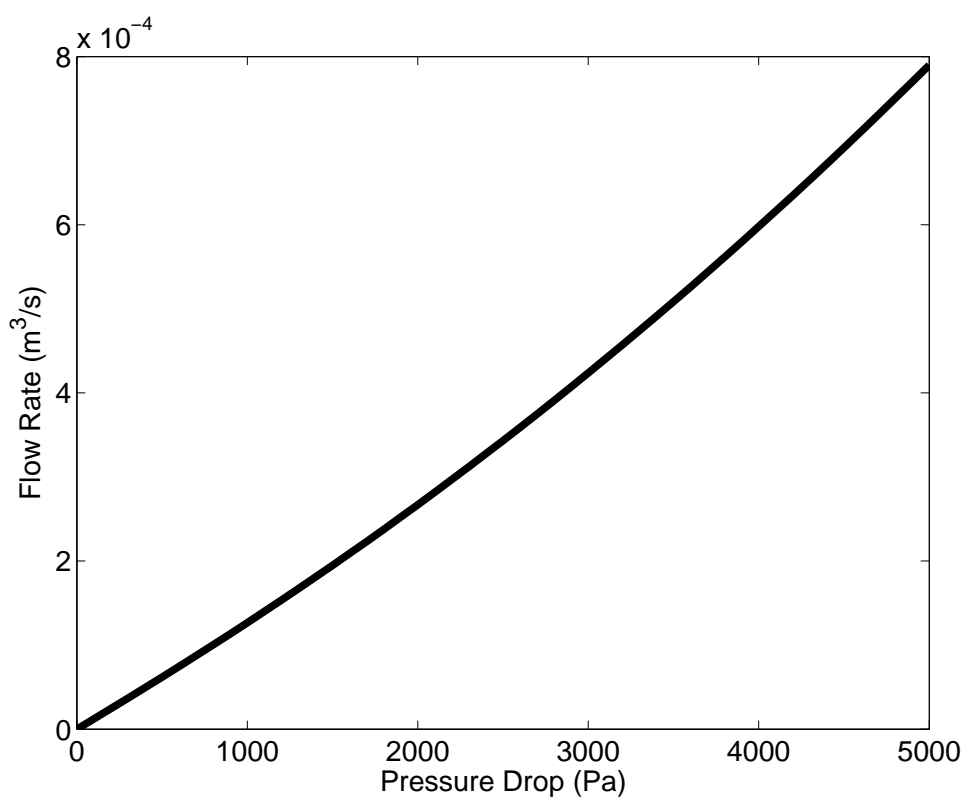

Figure 5: Flow rate versus pressure drop for the flow of a 6.0\% Elvanol 72-51 solution modeled by an Ellis fluid with $\mu_{o}=0.185 \mathrm{~Pa} . \mathrm{s}, \alpha=2.4$ and $\tau_{1 / 2}=1025 \mathrm{~Pa}$ flowing in a converging-diverging conic tube with $L=0.1 \mathrm{~m}, R_{m}=0.005 \mathrm{~m}$ and $R_{M}=0.02 \mathrm{~m}$. The bulk rheology of the fluid is taken from Sadowski dissertation [58]. 


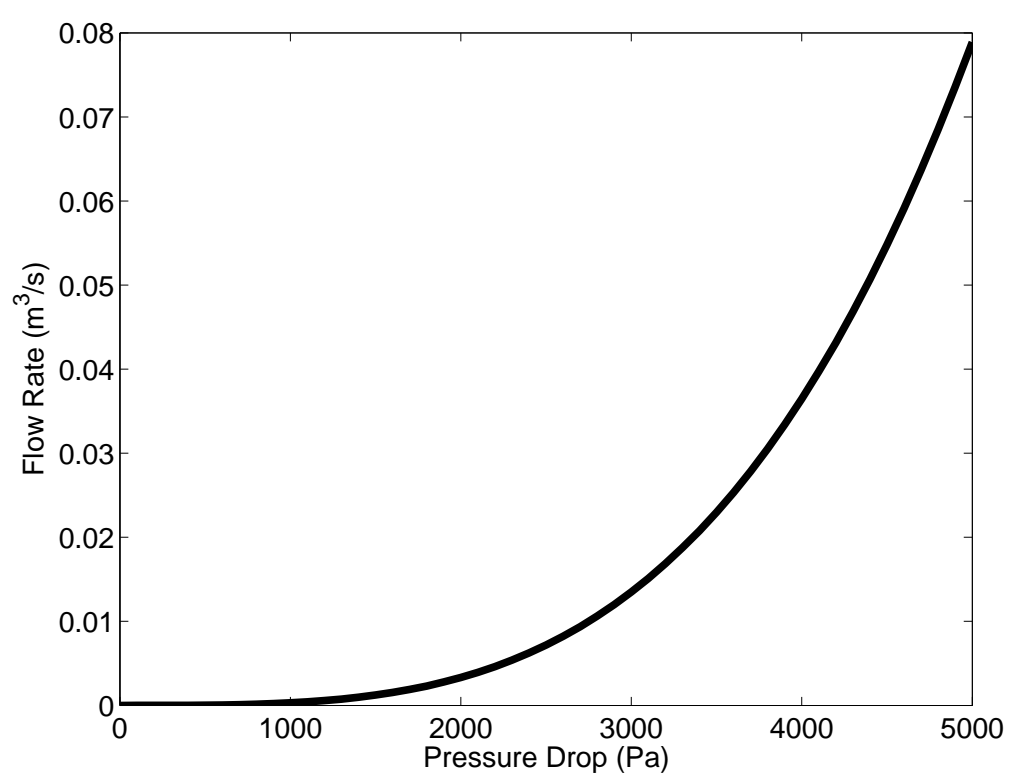

Figure 6: Flow rate versus pressure drop for the flow of a guar gum solution of $0.72 \%$ concentration modeled by an Ellis fluid with $\mu_{o}=2.672$ Pa.s, $\alpha=3.46$ and $\tau_{1 / 2}=9.01 \mathrm{~Pa}$ flowing in a converging-diverging parabolic tube with $L=0.06 \mathrm{~m}$, $R_{m}=0.005 \mathrm{~m}$ and $R_{M}=0.013 \mathrm{~m}$. The bulk rheology of the fluid is taken from Balhoff dissertation [21].

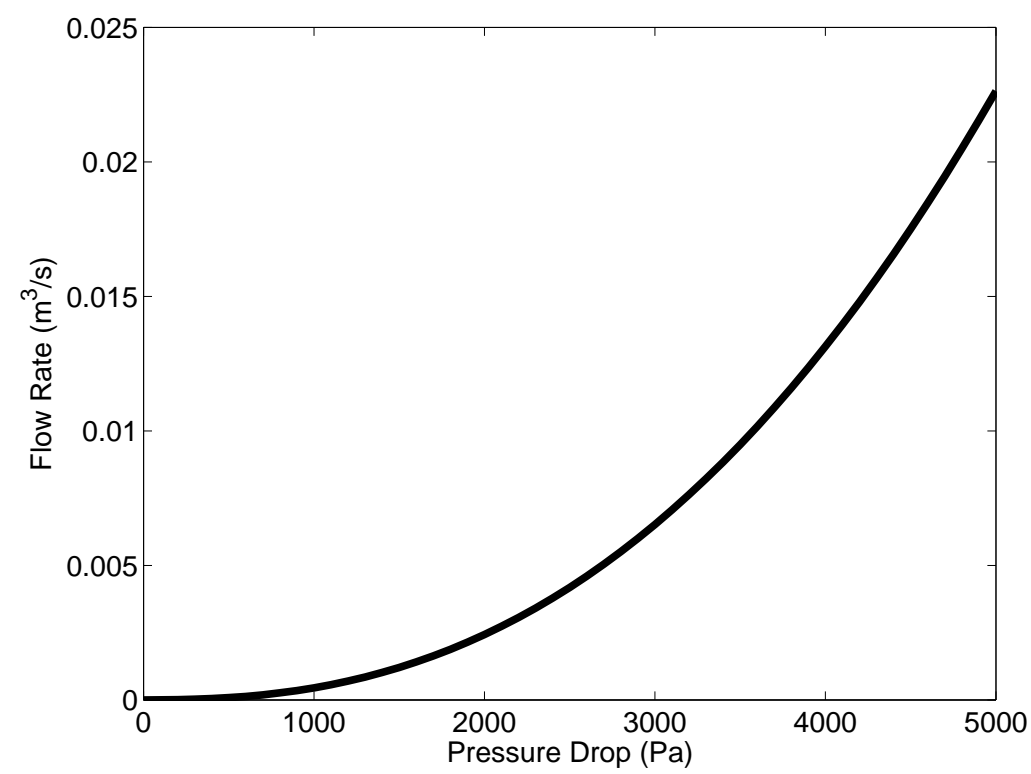

Figure 7: Flow rate versus pressure drop for the flow of an aqueous solution of polyacrylamide with $0.25 \%$ concentration modeled by an Ellis fluid with $\mu_{o}=$ 1.87862 Pa.s, $\alpha=2.4367$ and $\tau_{1 / 2}=0.5310 \mathrm{~Pa}$ flowing in a converging-diverging hyperbolic tube with $L=0.025 \mathrm{~m}, R_{m}=0.002 \mathrm{~m}$ and $R_{M}=0.006 \mathrm{~m}$. The bulk rheology of the fluid is taken from Park dissertation [59]. 


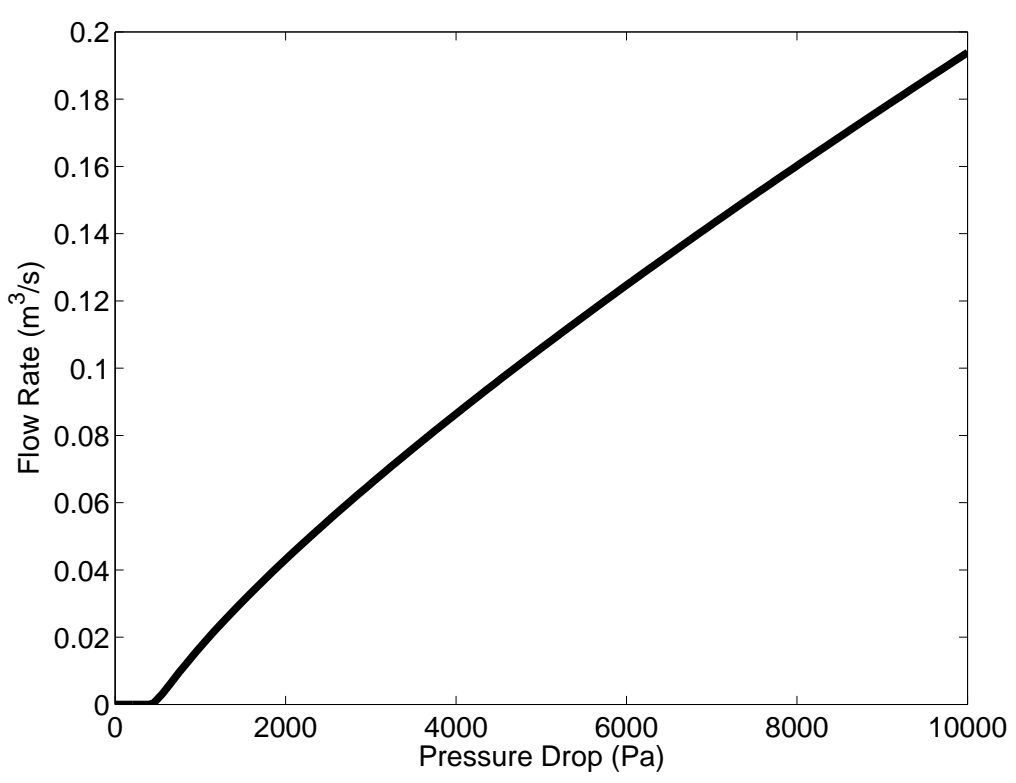

Figure 8: Flow rate versus pressure drop for the flow of a hypothetical shear thickening Herschel-Bulkley fluid with $C=0.075 \mathrm{~Pa} . \mathrm{s}^{n}, n=1.25$ and $\tau_{o}=20.0 \mathrm{~Pa}$ flowing in a converging-diverging hyperbolic cosine tube with $L=0.75 \mathrm{~m}, R_{m}=0.05 \mathrm{~m}$ and $R_{M}=0.15 \mathrm{~m}$. The threshold yield pressure is about $417 \mathrm{~Pa}$. Unlike the plots of shear-thinning and Bingham fluids, the curve concave downward due to the shear-thickening nature.

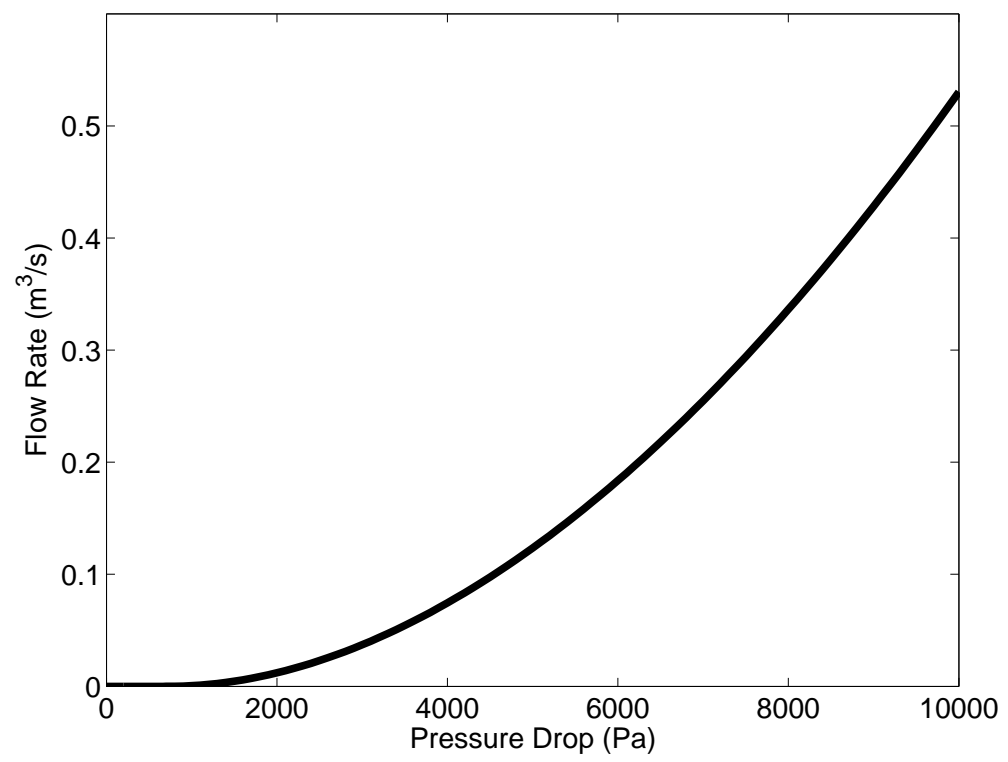

Figure 9: Flow rate versus pressure drop for the flow of a hypothetical shearthinning Herschel-Bulkley fluid with $C=0.673 \mathrm{~Pa}^{\mathrm{s}}{ }^{n}, n=0.54$ and $\tau_{o}=20.0 \mathrm{~Pa}$ flowing in a converging-diverging sinusoidal tube with $L=0.5 \mathrm{~m}, R_{m}=0.015 \mathrm{~m}$ and $R_{M}=0.06 \mathrm{~m}$. The threshold yield pressure is about $664 \mathrm{~Pa}$. 


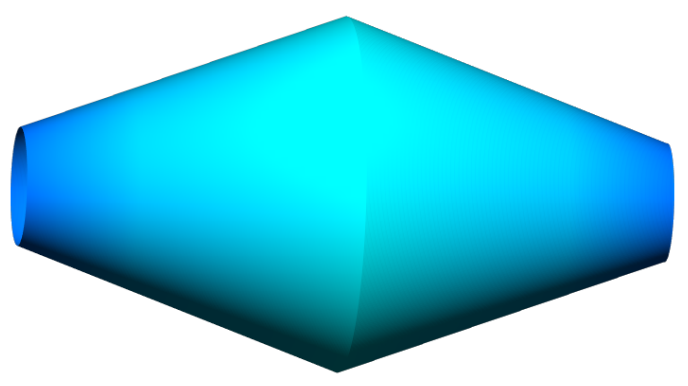

(a) Conic

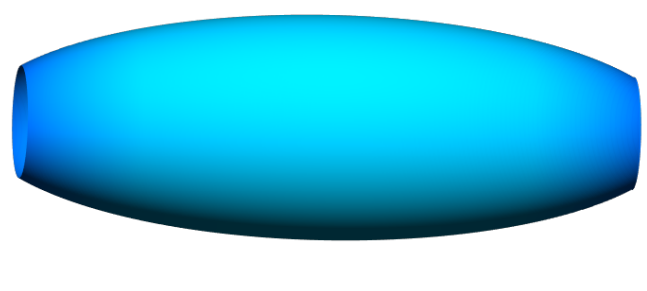

(b) Hyperbolic

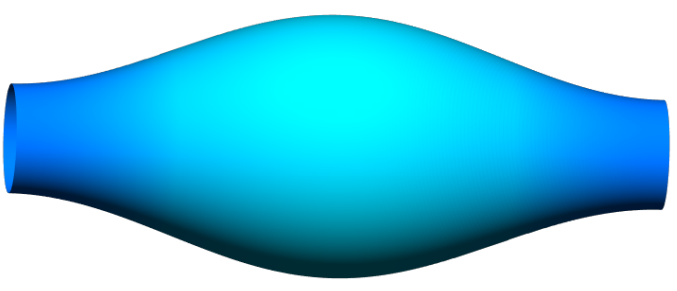

(c) Sinusoidal

Figure 10: A graphic demonstration of a sample of diverging-converging tube geometries.

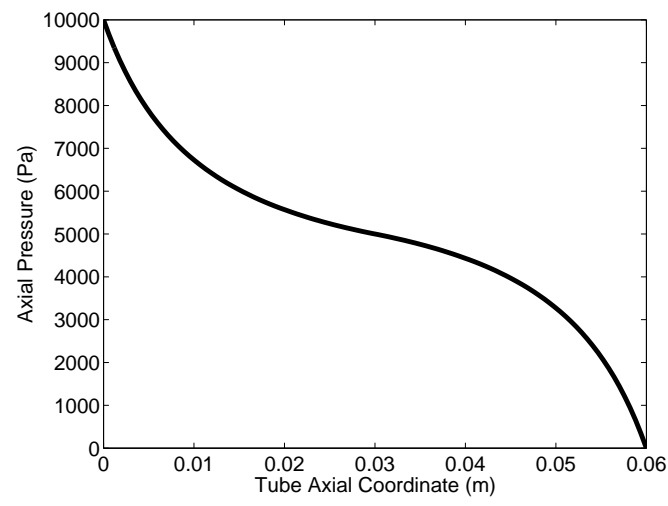

(a)

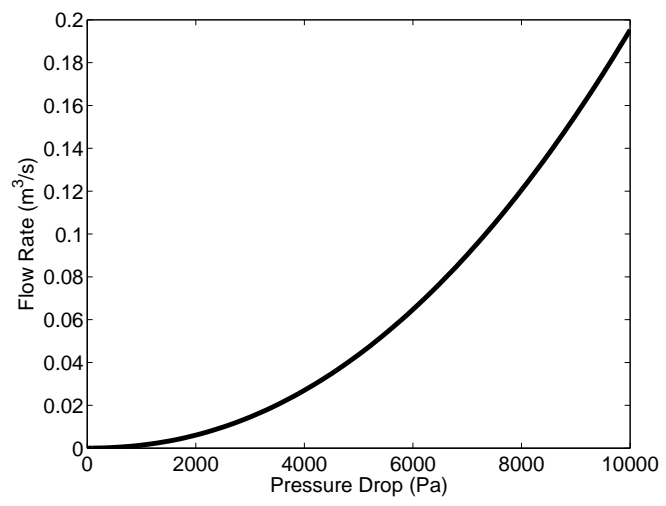

(b)

Figure 11: Simulated flow of $0.6 \%$ Natrosol - 250H solution modeled by an Ellis fluid with $\mu_{o}=0.4$ Pa.s, $\alpha=2.168$ and $\tau_{1 / 2}=5.2 \mathrm{~Pa}$ flowing in a diverging-converging conic tube, similar to the one in Figure 10 (a), with $L=0.06 \mathrm{~m}, R_{m}=0.005 \mathrm{~m}$ and $R_{M}=0.015 \mathrm{~m}$. The bulk rheology of the fluid is taken from Sadowski dissertation [58]. 


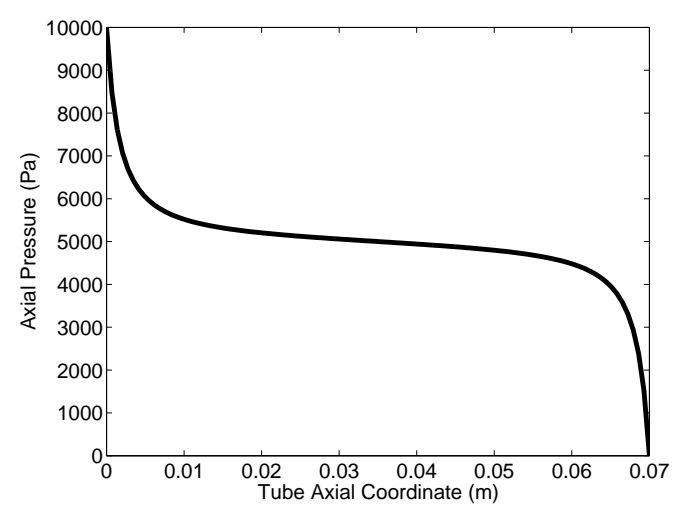

(a)

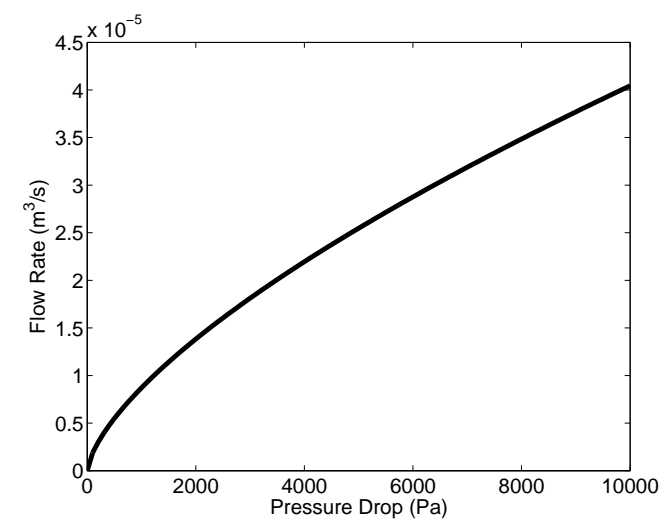

(b)

Figure 12: Simulated flow of a hypothetical shear-thickening power-law fluid with $C=0.75 \mathrm{~Pa} . \mathrm{s}^{n}$ and $n=1.5$ flowing in a diverging-converging hyperbolic tube, similar to the one in Figure $10(\mathrm{~b})$, with $L=0.07 \mathrm{~m}, R_{m}=0.005 \mathrm{~m}$ and $R_{M}=$ $0.013 \mathrm{~m}$. The flow rate plot concave downward due to the shear-thickening rheology.

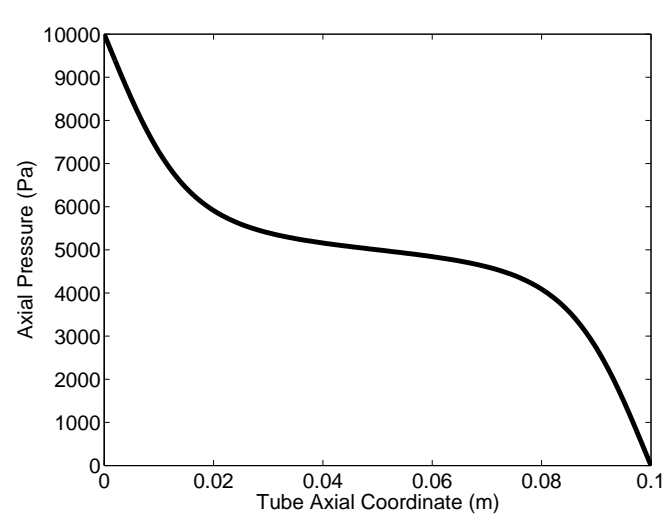

(a)

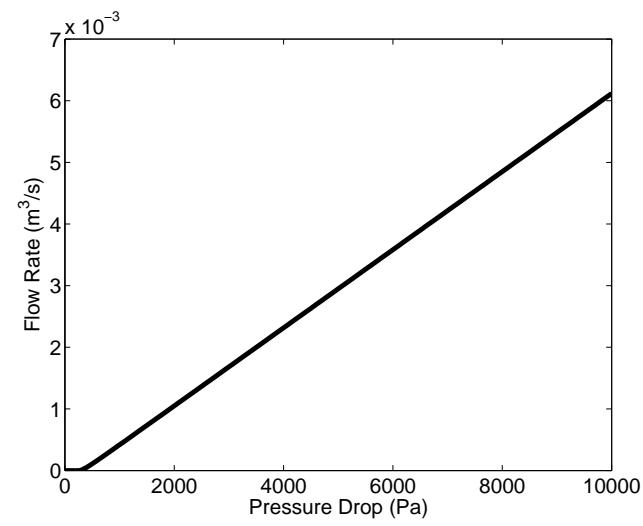

(b)

Figure 13: Simulated flow of an aqueous solution of Carbopol 941 with $1.0 \%$ concentration modeled by a Bingham fluid with $C=0.128$ Pa.s and $\tau_{o}=17.33 \mathrm{~Pa}$ flowing in a diverging-converging sinusoidal tube, similar to the one in Figure 10 (c), with $L=0.1 \mathrm{~m}, R_{m}=0.009 \mathrm{~m}$ and $R_{M}=0.02 \mathrm{~m}$. The threshold yield pressure is about $260 \mathrm{~Pa}$. The bulk rheology of the fluid is taken from Chase and Dachavijit [61]. 


\section{$5 \quad$ Tests and Validations}

Several consistency and validation tests have been carried out to verify the residualbased lubrication method and its code. In the following we outline these tests

- The sensibility of the method and the integrity of the code have been verified by smooth convergence to a final solution with mesh refinement by employing finer discretization.

- Tests have been carried out on the flow of non-Newtonian fluids through straight cylindrical tubes with constant radii as a special case for the convergingdiverging tubes. In all cases the numerical solutions converged to the correct analytical solutions as given by Equations 2 and 4 .

- The method and the code have also been verified by the convergence to the correct analytical solutions for the flow of Newtonian fluids, as a special case for the non-Newtonian fluids, in the five prototype converging-diverging geometries. These geometries have analytical solutions, given in Table 4, that have been derived and validated previously for the flow of Newtonian fluids $[38,40,42]$. The Newtonian numerical solutions have been obtained both as Poiseuille flow and as Herschel-Bulkley flow with $\tau_{o}=0$ and $n=1$; both of these numerical solutions were identical to the corresponding analytical solution within acceptable numerical errors.

- The convergence to the correct analytical solution has also been verified for the flow of non-Newtonian power-law fluids through these five convergingdiverging geometries using analytical expressions that have been derived and validated previously in [41]; these analytical expressions are given in Table 5. A sample of these comparisons between the numerical and analytical solutions is given in Figure 14 for the flow of a typical power-law fluid through a tube 
with a conic geometry. As seen, the two solutions are virtually identical within acceptable numerical errors.

Table 4: The equations describing the dependency of the flow rate $Q$ on the pressure drop $\Delta p$ for the flow of Newtonian fluids through rigid tubes with the five converging-diverging geometries of Table 1 . These equations were derived and validated previously in [42].

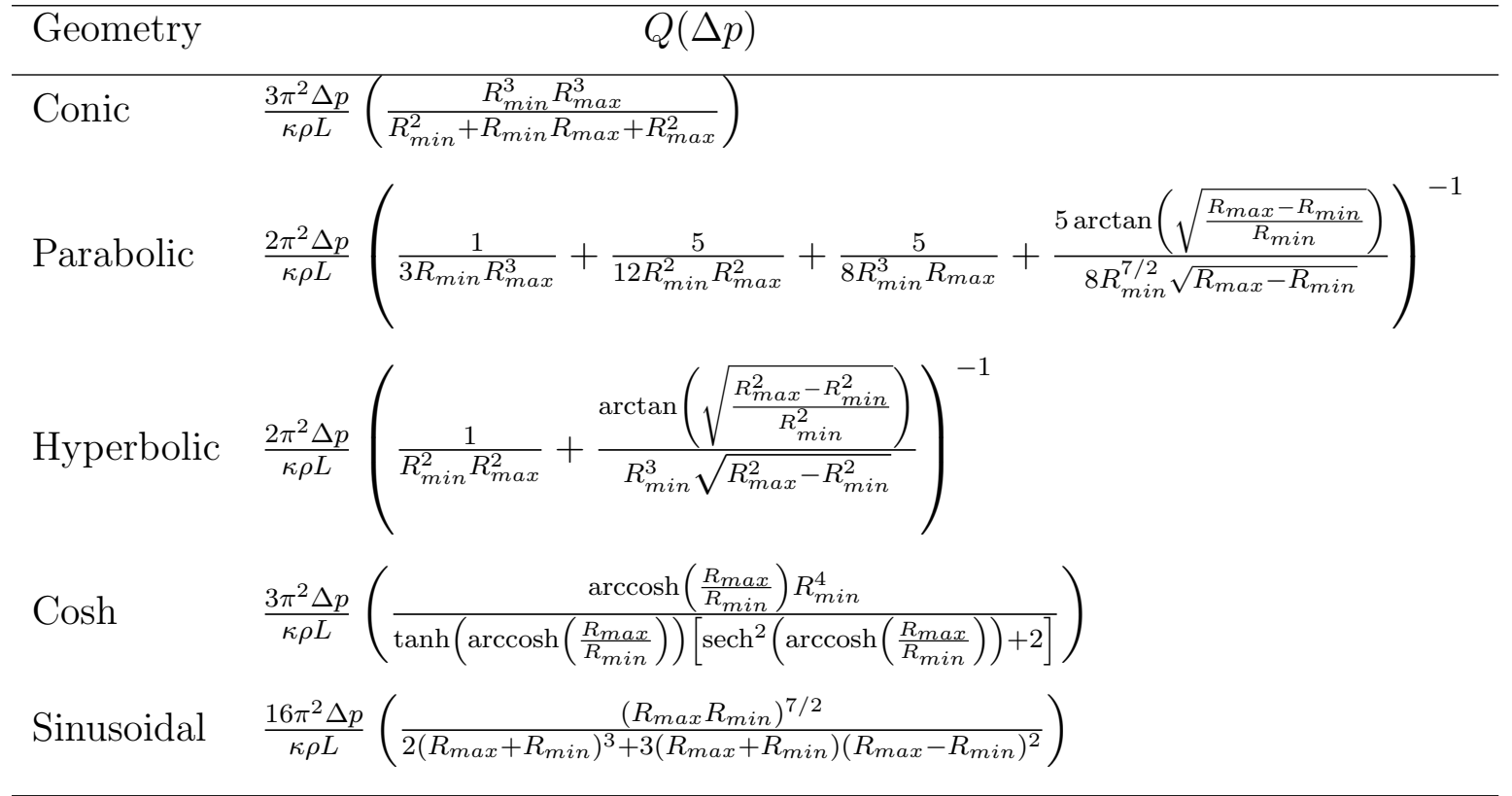

Table 5: The equations describing the dependency of the flow rate $Q$ on the pressure drop $\Delta p$ for the flow of power-law fluids in rigid tubes for the five convergingdiverging geometries of Table 1 where $F_{1}$ is the Appell hypergeometric function, ${ }_{2} F_{1}$ is the hypergeometric function and $\mathrm{Im}$ is the imaginary part of the given function. These relations were previously [41] derived and validated.

\begin{tabular}{ll}
\hline Geometry & \\
\hline Conic & {$\left[\frac{3 \pi^{n} n^{n+1}\left(R_{M}-R_{m}\right) \Delta p}{2 L C(3 n+1)^{n}}\left(\frac{R_{m}^{3 n} R_{M}^{3 n}}{R_{M}^{3 n}-R_{m}^{3 n}}\right)\right]^{1 / n}$} \\
Parabolic & {$\left[\frac{n^{n} \pi^{n} R_{m}^{3 n+1} \Delta p}{2 L C(3 n+1)^{n}{ }_{2} F_{1}\left(\frac{1}{2}, 3 n+1 ; \frac{3}{2} ; 1-\frac{R_{M}}{R_{m}}\right)}\right]^{1 / n}$} \\
Hyperbolic & {$\left[\frac{\pi^{n} n^{n} R_{m}^{3 n+1} \Delta p}{2 L C(3 n+1)^{n}{ }_{2} F_{1}\left(\frac{1}{2}, \frac{3 n+1}{2} ; \frac{3}{2} ; 1-\frac{R_{M}^{2}}{R_{m}^{2}}\right)}\right]^{1 / n}$} \\
Hyperbolic Cosine & {$\left[\frac{3 \pi^{n} n^{n+1} R_{m} R_{M}^{3 n} \operatorname{arccosh}\left(\frac{R_{M}}{R_{m}}\right) \Delta p}{2 L C(3 n+1)^{n} \operatorname{Im}\left({ }_{2} F_{1}\left(\frac{1}{2},-\frac{3 n}{2} ; \frac{2-3 n}{2} ; \frac{R_{M}^{2}}{R_{m}^{2}}\right)\right)}\right]^{1 / n}$} \\
Sinusoidal & {$\left[\frac{3 \pi^{n+1} n^{n+1} R_{M}^{3 n} \sqrt{R_{M} R_{m}} \Delta p}{2 L C(3 n+1)^{n} \operatorname{Im}\left(F_{1}\left(-3 n ; \frac{1}{2}, \frac{1}{2} ; 1-3 n ; 1, \frac{R_{M}}{R_{m}}\right)\right)}\right]^{1 / n}$}
\end{tabular}




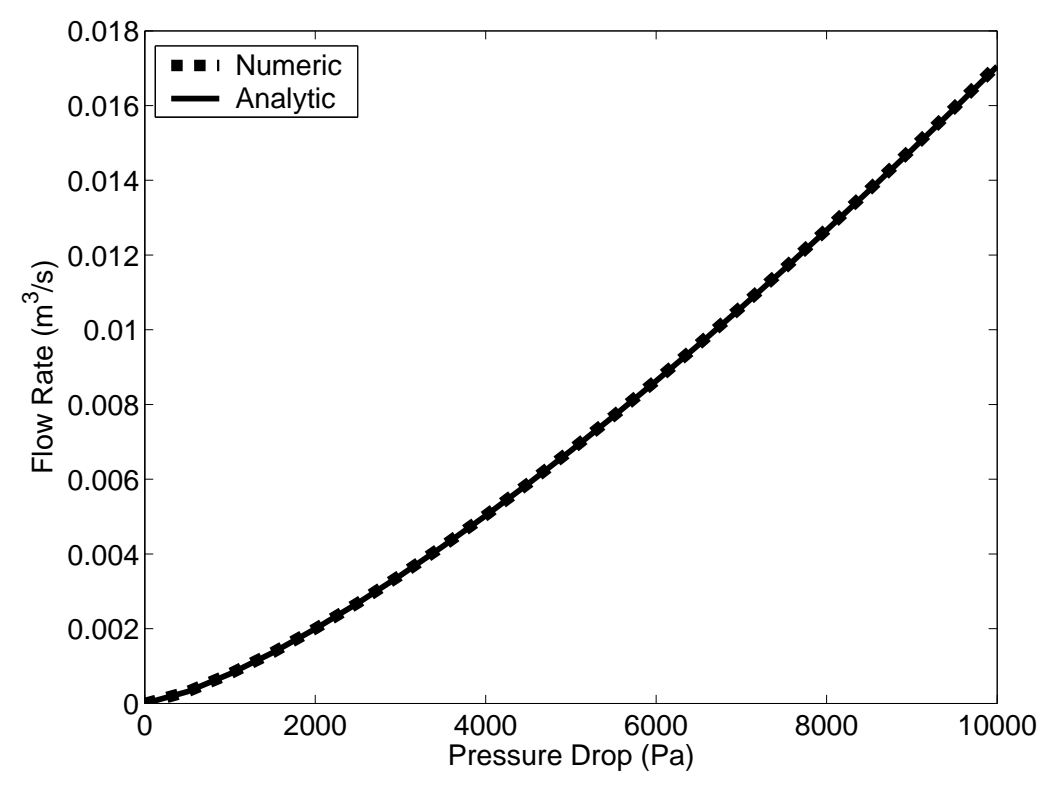

Figure 14: Comparing the numerical and analytical solutions for the flow of a typical power-law fluid with $C=0.5 \mathrm{~Pa} . \mathrm{s}^{n}$ and $n=0.75$ through a conically-shaped converging-diverging tube with $L=0.15 \mathrm{~m}, R_{m}=0.01 \mathrm{~m}$ and $R_{M}=0.02 \mathrm{~m}$. The analytical solution is obtained from the first equation in Table 5. 


\section{Computational Issues and Comparison}

Apart from yield-stress fluids, we did not experience convergence difficulties that normally associate such non-linear solution schemes like those that we confronted in our previous investigation to the flow of fluids through distensible structures $[38,57]$. In almost all the cases investigated in the current study, the convergence was immediate as it occurred within a few Newton-Raphson iterations even for the high-pressure flow regime cases with the use of extreme boundary conditions and eccentric parameters for the flow, fluids and tube geometry.

As for the yield-stress fluids, there were some convergence difficulties and hence to overcome these difficulties we used a number of numerical tricks. The most effective of these tricks may be to start with solving the problem assuming zero yield-stress. We then use this yield-free solution as an initial guess for solving the initial problem with non-zero yield-stress. The final flow solution in the yieldstress fluid cases is conditioned that if it resulted in $\tau_{o}>\tau_{w}$ on any element, the flow in the tube is set to zero. It should be remarked that for the flow of yieldstress fluids through cylindrical tubes with fixed radii, the yield condition where the pressure drop just overcomes the yield-stress and hence the flow starts is given by the following relation $[22,52,62]$

$$
\tau_{w}>\tau_{o} \quad \Longrightarrow \quad \Delta p>\frac{2 L \tau_{o}}{R}
$$

For the high-pressure flow regimes, the convergence is usually more difficult than for the low-pressure flow regimes, especially for yield-stress fluids. An effective trick in these cases is to step up on the pressure ladder by starting the process with solving the same problem but with low pressure boundary conditions where the convergence is easy. This low-pressure solution is then used as an initial guess for the next step with a higher boundary pressure. On repeating this process with the 
use of a suitably divided pressure field, e.g. 100 Pa increase per step, high-pressure flow regime problems can be solved without convergence difficulties. Although this stepping up process incurs an extra computational cost, in most cases this extra cost is very low.

Also for the yield-stress fluids, the access of the flow regimes at the border of the threshold yield pressure, which is required for determining the exact yield point, may not be easy due to the absence of solutions before yield with possible difficulties in providing a sensible initial guess. The trick then is to start from a relatively high-pressure flow regime where the convergence to a solution is easy and where the flow system is expected to have yielded already. The solution at this regime is then used as an initial guess for stepping down on the pressure ladder, as for stepping up process which is outlined earlier. By using small pressure steps, e.g. $1 \mathrm{~Pa}$ decrease per step, the flow regimes at the very edge of the threshold yield pressure can be accessed and hence the exact threshold yield pressure can be determined.

Regarding the CPU time and memory requirements, the residual-based method has a very low computational cost. This is partly due to the nature of the problem which is related to single tubes and hence it is normally of limited size. The discretization usually involves a few tens or at most a few hundreds of elements on the discretization meshes. Hence the computational cost normally does not exceed a few kilobytes of memory and a few seconds of CPU time on a normal computer.

The advantages of the residual-based method over the traditional methods is simplicity, generality, ease of implementation, very good rate and speed of convergence, and very low computational cost. Moreover, the solutions obtained by the residual-based method match in their accuracy any existing or anticipated analytical solutions within the given physical and numerical approximations. The accuracy of the residual-based method and its convergence to the correct analyti- 
cal solutions are confirmed in all cases in which analytical solutions are available such as the limiting cases of straight geometries and Newtonian fluids, as well as the available analytical solutions for the non-Newtonian power-law fluids as given by the equations in Table 5 . The main disadvantage of the residual-based method is its one-dimensional nature and hence it cannot be used for obtaining the parameters of the flow field in dimensions other than the axial direction. The method has also other limitations related to the physical assumptions on which the underlying flow model is based. However, most of these limitations are shared by other comparable methods.

\section{Conclusions}

The investigation in this paper led to the proposal, formulation and validation of a residual-based lubrication method that can be used to obtain the pressure field and flow rate in rigid tubes with converging and diverging features, or more generally with a cross section that vary in size and/or shape in the flow direction, for the flow of non-Newtonian fluids.

The method in its current formulation can be applied to the entire category of time-independent non-Newtonian fluids, that is generalized Newtonian fluids. Two time-independent non-Newtonian fluids, namely Ellis and Herschel-Bulkley, have been used in this investigation. The method, in our judgement, has the capability to be extended to the history-dependent non-Newtonian fluids, i.e. viscoelastic and thixotropic/rheopectic.

Five converging-diverging and diverging-converging geometries have been used for test, validation, demonstration and as prototypes for the investigation of such flows. The method, however, has a wider applicability range with regard to the tube geometry as it can be applied to all geometries that vary in size and/or shape in the flow direction as long as a characteristic relation, analytical or empirical or 
numerical, that correlates the flow rate to the pressure drop over the presumablystraight discretized elements can be found.

The residual-based lubrication method was validated by the convergence to the correct analytical solutions in the special cases of straight constant-radius geometries and Newtonian fluids, as well as the convergence to the analytical solutions for the flow of power-law fluids through the five converging-diverging prototype geometries. The method has also been endorsed by a number of qualitativelysensible tendencies such as the convergence to a final solution with improved mesh refinement.

The residual-based method has obvious advantages as compared to the more traditional methods. These advantages include generality, ease of implementation, low computational cost, good rate and speed of convergence, reliability, and accuracy. The main limitation of the method is its one-dimensional nature. 


\section{Nomenclature}

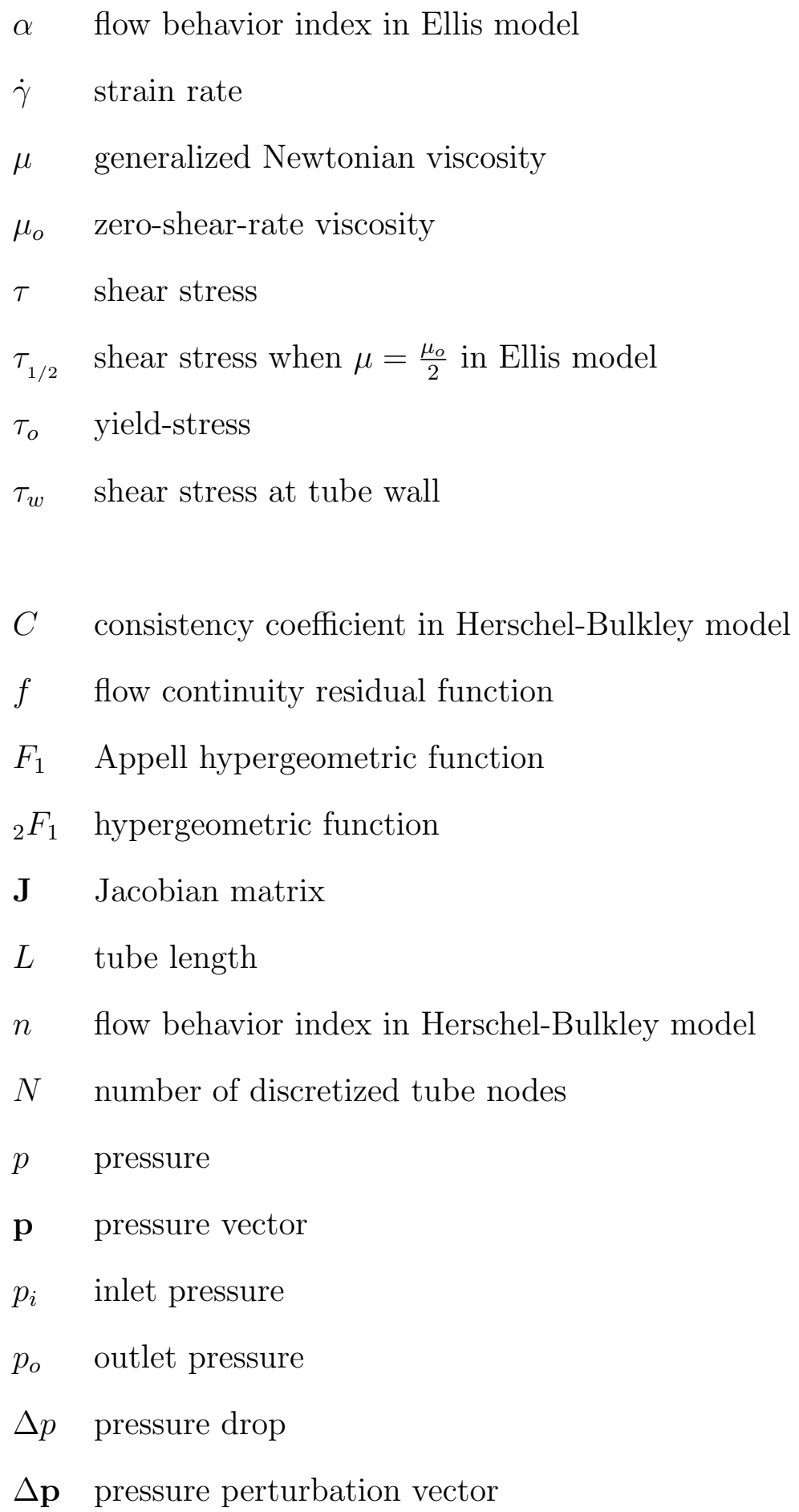


$Q \quad$ volumetric flow rate

$Q_{E} \quad$ numeric flow rate for Ellis model

$Q_{H} \quad$ numeric flow rate for Herschel-Bulkley model

$Q_{P} \quad$ numeric flow rate for Poiseuille model

r residual vector

$R \quad$ tube radius

$R_{m}$ minimum radius of converging-diverging tube

$R_{M}$ maximum radius of converging-diverging tube

$x \quad$ tube axial coordinate 


\section{References}

[1] E.W. Williams; S.H. Javadpour. The flow of an elastico-viscous liquid in an axisymmetric pipe of slowly varying cross-section. Journal of Non-Newtonian Fluid Mechanics, 7(2-3):171-188, 1980. 4

[2] A. Hooper; B.R. Duffy; H.K. Moffatt. Flow of fluid of non-uniform viscosity in converging and diverging channels. Journal of Fluid Mechanics, 117:283-304, 1982. 4

[3] T. Ishikawa; L.F.R. Guimaraes; S. Oshima; R. Yamane. Effect of nonNewtonian property of blood on flow through a stenosed tube. Fluid Dynamics Research, 22:251-264, 1998. 4

[4] P.K. Mandal. An unsteady analysis of non-Newtonian blood flow through tapered arteries with a stenosis. International Journal of Non-Linear Mechanics, 40(1):151-164, 2005. 4

[5] A. Valencia; A. Zarate; M. Galvez; L. Badilla. Non-Newtonian blood flow dynamics in a right internal carotid artery with a saccular aneurysm. International Journal for Numerical Methods in Fluids, 50(6):751-764, 2006. 4

[6] C. Fisher; J.S. Rossmann. Effect of Non-Newtonian Behavior on Hemodynamics of Cerebral Aneurysms. Journal of Biomechanical Engineering, 131(9):091004(1-9), 2009. 4

[7] J.C. Misra; S. Maiti. Peristaltic Pumping of Blood Through Small Vessels of Varying Cross-Section. Journal of Applied Mechanics, 79(6):061003-061021, 2012. 4

[8] T. Sochi. Non-Newtonian Rheology in Blood Circulation. Submitted, 2013. 4 
[9] D.A. White. Non-Newtonian flow in stratified porous media and in axisymmetric geometries. Chemical Engineering Science, 23(3):243-251, 1968. 4

[10] N. Phan-Thien; C.J. Goh; M.B. Bush. Viscous flow through corrugated tube by boundary element method. Journal of Applied Mathematics and Physics (ZAMP), 36(3):475-480, 1985. 4

[11] N. Phan-Thien; M.M.K. Khan. Flow of an Oldroyd-type fluid through a sinusoidally corrugated tube. Journal of Non-Newtonian Fluid Mechanics, 24(2):203-220, 1987. 4

[12] D.F. James; N. Phan-Thien; M.M.K. Khan; A.N. Beris; S. Pilitsis. Flow of test fluid M1 in corrugated tubes. Journal of Non-Newtonian Fluid Mechanics, $35(2-3): 405-412,1990.4$

[13] A. Souvaliotis; A.N. Beris. Applications of domain decomposition spectral collocation methods in viscoelastic flows through model porous media. Journal of Rheology, 36(7):1417-1453, 1992. 4

[14] D.W. Ruth; H. Ma. Numerical analysis of viscous, incompressible flow in a diverging-converging RUC. Transport in Porous Media, 13(2):161-177, 1993. 4

[15] A. Souvaliotis; A.N. Beris. Spectral collocation/domain decomposition method for viscoelastic flow simulations in model porous geometries. Computer Methods in Applied Mechanics and Engineering, 129(1):9-28, 1996. 4

[16] W.G. Gray; C.T. Miller. Examination of Darcy's Law for Flow in Porous Media with Variable Porosity. Environmental Science \& Technology, 38(22):5895$5901,2004.4$

[17] T. Sochi. Computational Techniques for Modeling Non-Newtonian Flow in 
Porous Media. International Journal of Modeling, Simulation, and Scientific Computing, 1(2):239-256, 2010. 4

[18] T. Sochi. Non-Newtonian Flow in Porous Media. Polymer, 51(22):5007-5023, 2010. 4

[19] P.H. Valvatne. Predictive pore-scale modelling of multiphase flow. PhD thesis, Imperial College London, 2004. 4

[20] X. Lopez. Pore-scale modelling of non-Newtonian flow. PhD thesis, Imperial College London, 2004. 4

[21] M.T. Balhoff. Modeling the flow of non-Newtonian fluids in packed beds at the pore scale. $\mathrm{PhD}$ thesis, Louisiana State University, 2005. 4, 17

[22] T. Sochi. Pore-Scale Modeling of Non-Newtonian Flow in Porous Media. PhD thesis, Imperial College London, 2007. 4, 6, 7, 8, 24

[23] R. Xu; S. Luo; P. Jiang. Pore scale numerical simulation of supercritical $\mathrm{CO}_{2}$ injecting into porous media containing water. Energy Procedia, 4:4418-4424, 2011. 4

[24] T. Tosco; D.L. Marchisio; F. Lince; R. Sethi. Extension of the DarcyForchheimer Law for Shear-Thinning Fluids and Validation via Pore-Scale Flow Simulations. Transport in Porous Media, 96(1):1-20, 2013. 4

[25] T. Sochi. Pore-scale modeling of viscoelastic flow in porous media using a Bautista-Manero fluid. International Journal of Heat and Fluid Flow, 30(6):1202-1217, 2009. 4, 5

[26] V. Di Federico; M. Pinelli; R. Ugarelli. Estimates of effective permeability for non-Newtonian fluid flow in randomly heterogeneous porous media. Stochastic Environmental Research and Risk Assessment, 24(7):1067-1076, 2010. 4 
[27] A. Afsharpoor; M.T. Balhoff; R. Bonnecaze; C. Huh. CFD modeling of the effect of polymer elasticity on residual oil saturation at the pore-scale. Journal of Petroleum Science and Engineering, 94-95:79-88, 2012. 4

[28] W. Liu; J. Yao; Y. Wang. Exact analytical solutions of moving boundary problems of one-dimensional flow in semi-infinite long porous media with threshold pressure gradient. International Journal of Heat and Mass Transfer, 55(2122):6017-6022, 2012. 4

[29] F.J. Galindo-Rosales; L. Campo-Deaño; F.T. Pinho; E. van Bokhorst; P.J. Hamersma; M.S.N. Oliveira; M.A. Alves. Microfluidic systems for the analysis of viscoelastic fluid flow phenomena in porous media. Microfluidics and Nanofluidics, 12(1-4):485-498, 2012. 4

[30] L. Campo-Deaño; F.J. Galindo-Rosales; F.T. Pinho; M.A. Alves; M.S.N. Oliveira. Nanogel formation of polymer solutions flowing through porous media. Soft Matter, 8:6445-6453, 2012. 4

[31] S. Wang; A.F. Clarens. The effects of $\mathrm{CO}_{2}$-brine rheology on leakage processes in geologic carbon sequestration. Water Resources Research, 48(8):W08518, 2012. 4

[32] V. Ciriello; V. Di Federico. Similarity solutions for flow of non-Newtonian fluids in porous media revisited under parameter uncertainty. Advances in Water Resources, 43:3851, 2012. 4

[33] T. Chevalier; C. Chevalier; X. Clain; J.C. Dupla; J. Canou; S. Rodts; P. Coussot. Darcy's law for yield stress fluid flowing through a porous medium. Journal of Non-Newtonian Fluid Mechanics, 195:5766, 2013. 4

[34] A. Afsharpoor; M.T. Balhoff. Static and Dynamic CFD Modeling of Viscoelastic Polymer: Trapped Oil Displacement and Deformation at the Pore-Level. 
SPE Annual Technical Conference and Exhibition, 30 September - 2 October 2013, New Orleans, Louisiana, USA, 2013. 4

[35] H.O. Balan. Dynamics of Foam Mobility in Porous Media. PhD thesis, University of Texas at Austin, 2013. 4

[36] K.K. Farayola; A.T. Olaoye; A. Adewuyi. Petroleum Reservoir Characterisation for Fluid with Yield Stress Using Finite Element Analyses. Nigeria Annual International Conference and Exhibition, 30 July - 1 August 2013, Lagos, Nigeria, 2013. 4

[37] S.R. Burdette; P.J. Coates; R.C. Armstrong; R.A. Brown. Calculations of viscoelastic flow through an axisymmetric corrugated tube using the explicitly elliptic momentum equation formulation (EEME). Journal of Non-Newtonian Fluid Mechanics, 33(1):1-23, 1989. 4

[38] T. Sochi. Flow of Navier-Stokes Fluids in Converging-Diverging Distensible Tubes. Submitted, 2013. 4, 9, 21, 24

[39] S.C. Fu; W.W.F. Leung; R.M.C. So. A lattice Boltzmann and immersed boundary scheme for model blood flow in constricted pipes: Part 1 - steady flow. Communications in Computational Physics, 14(1):126-152, 2013. 4

[40] T. Sochi. The Flow of Newtonian Fluids in Axisymmetric Corrugated Tubes. arXiv:1006.1515v1, 2010. 4, 21

[41] T. Sochi. The flow of power-law fluids in axisymmetric corrugated tubes. Journal of Petroleum Science and Engineering, 78(3-4):582-585, 2011. 4, 21, 22

[42] T. Sochi. Newtonian Flow in Converging-Diverging Capillaries. International Journal of Modeling, Simulation, and Scientific Computing, 04(03):1350011, 2013. $4,21,22$ 
[43] M.T. Balhoff; K.E. Thompson. A macroscopic model for shear-thinning flow in packed beds based on network modeling. Chemical Engineering Science, 61(2):698-719, 2006. 5

[44] H.O. Balan; M.T. Balhoff; Q.P. Nguyen; W.R. Rossen. Network Modeling of Gas Trapping and Mobility in Foam Enhanced Oil Recovery. Energy \& Fuels, 25(9):3974-3987, 2011. 5

[45] T. Sochi. Using Euler-Lagrange Variational Principle to Obtain Flow Relations for Generalized Newtonian Fluids. Rheologica Acta (accepted), 2013. 5

[46] T.J. Sadowski; R.B. Bird. Non-Newtonian flow through porous media I. Theoretical. Transactions of the Society of Rheology, 9(2):243-250, 1965. 6

[47] J.G. Savins. Non-Newtonian Flow Through Porous Media. Industrial and Engineering Chemistry, 61(10):18-47, 1969. 6

[48] R.B. Bird; R.C. Armstrong; O. Hassager. Dynamics of Polymeric Liquids, volume 1. John Wily \& Sons, second edition, 1987. 6

[49] P.J. Carreau; D. De Kee; R.P. Chhabra. Rheology of Polymeric Systems. Hanser Publishers, 1997. 6

[50] T. Sochi. Flow of Non-Newtonian Fluids in Porous Media. Journal of Polymer Science Part B, 48(23):2437-2467, 2010. 6, 7

[51] T. Sochi. Slip at Fluid-Solid Interface. Polymer Reviews, 51:1-33, 2011. 6, 8

[52] T. Sochi; M.J. Blunt. Pore-scale network modeling of Ellis and HerschelBulkley fluids. Journal of Petroleum Science and Engineering, 60(2):105-124, 2008. $7,8,24$

[53] A.H.P. Skelland. Non-Newtonian Flow and Heat Transfer. John Wiley and Sons Inc., 1967. 7, 8 
[54] S. Liu; J.H. Masliyah. On non-Newtonian fluid flow in ducts and porous media - Optical rheometry in opposed jets and flow through porous media. Chemical Engineering Science, 53(6):1175-1201, 1998. 7

[55] Y. Damianou; G.C. Georgiou; I. Moulitsas;. Combined effects of compressibility and slip in flows of a Herschel-Bulkley fluid. Journal of Non-Newtonian Fluid Mechanics, 193:89-102, 2013. 8

[56] T. Sochi. One-Dimensional Navier-Stokes Finite Element Flow Model. arXiv:1304.2320, 2013. 9

[57] T. Sochi. Pore-Scale Modeling of Navier-Stokes Flow in Distensible Networks and Porous Media. Submitted, 2013. 9, 24

[58] T.J. Sadowski. Non-Newtonian flow through porous media. PhD thesis, University of Wisconsin, 1963. 13, 16, 19

[59] H.C. Park. The flow of non-Newtonian fluids through porous media. PhD thesis, Michigan State University, 1972. 13, 16, 17

[60] T.F. Al-Fariss; K.L. Pinder. Flow of a shear-thinning liquid with yield stress through porous media. SPE 13840, 1984. 16

[61] G.G. Chase; P. Dachavijit. Incompressible cake filtration of a yield stress fluid. Separation Science and Technology, 38(4):745-766, 2003. 16, 20

[62] T. Sochi. Modelling the Flow of Yield-Stress Fluids in Porous Media. Transport in Porous Media, 85(2):489-503, 2010. 24 